\title{
New WHO guidelines on intimate-partner violence
}

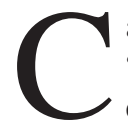

alling violence against women "a global health problem of epidemic proportions," the World Health Organization (WHO) has issued new practice and policy guidelines to help health care practitioners screen, treat and support victims of sexual and partner violence.

The new guidelines discourage universal screening, on the basis that it has not produced better outcomes for women. Instead, they ask doctors to inquire about intimate-partner violence when assessing conditions that could be caused or complicated by such violence. Those conditions include anxiety, depression, posttraumatic stress disorder, sleep disorders, suicidality or other forms of self-harm, alcohol and other substance use and unexplained reproductive or gastrointestinal symptoms, among other symptoms the guidelines list.

The guidelines are "the clearest statement to date on the importance of a careful approach to talking to women about exposure to violence, and using identification measures that are grounded in that clinical encounter," says Nadine Wathen, an associate professor and violence researcher at Western University in London, Ontario, who was an external reviewer of the guidelines.

Instead of reading a rote list of questions, as some doctors in California, Virginia and Pennsylvania are required to do because of legislation mandating screening, the guidelines encourage a more personal encounter, based on clinical flags that signal a woman's potential exposure to violence, says Wathen. "Ask her how she is doing. Ask her if she is safe at home - and then start that conversation. Her safety and feeling of support is paramount in that process."

The guidelines emphasize the value of providing nonjudgmental, validating support, as well as practical care. They urge doctors and other health care practitioners to provide or mobilize social

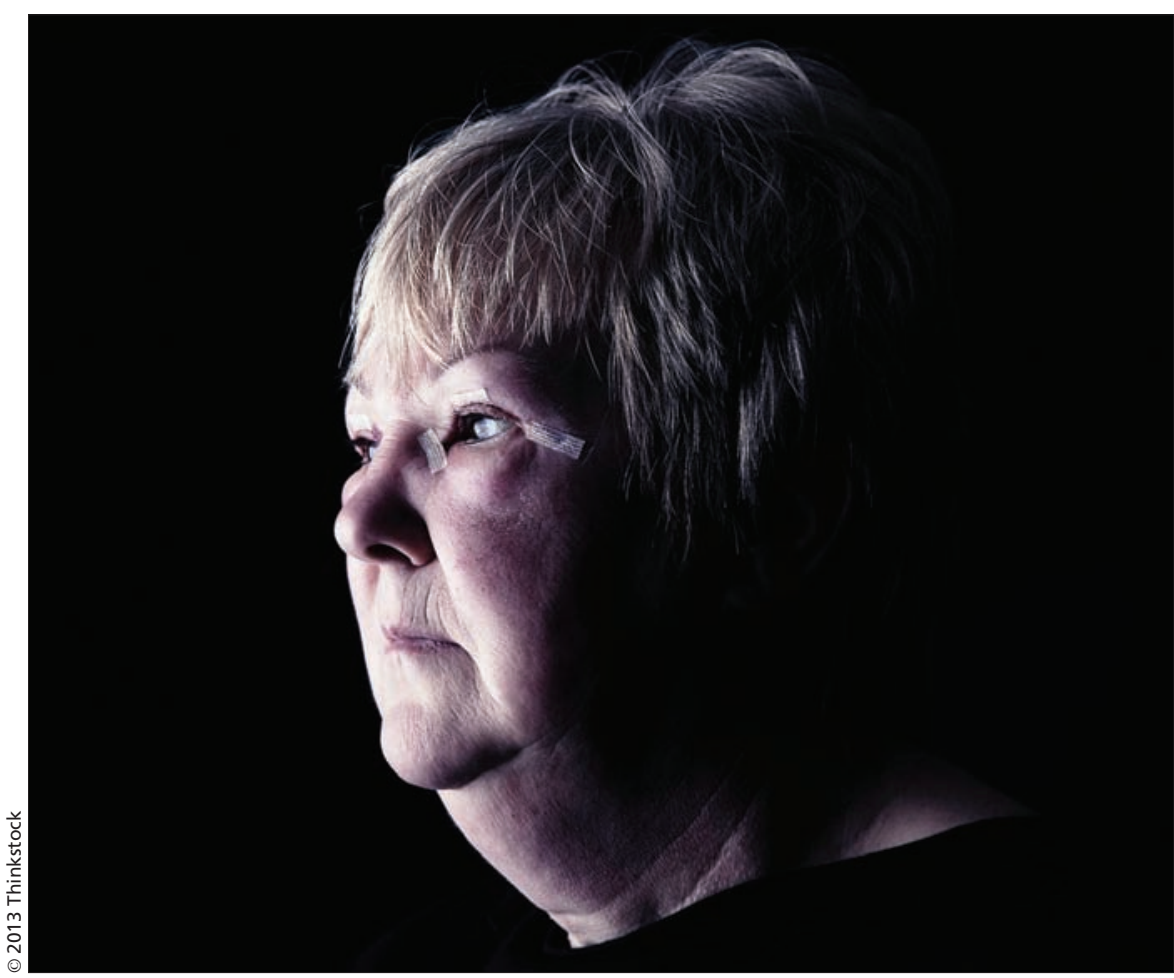

New WHO guidelines discourage universal screening for intimate-partner violence, recommending instead that doctors screen for potential victims when assessing conditions that could be caused or complicated by such violence.

support and access to legal assistance, as well as to help women increase their own safety and that of their children.

In addition, survivors of violence should be offered one of two specific therapies to reduce posttraumatic stress: cognitive behavior therapy or eye movement desensitization and reprocessing, the guidelines state. Accessing these therapies remains difficult, however, because they are largely delivered by psychologists, social workers and other mental health therapists and are not covered by provincial health care plans, says Dr. Barbara Lent, a professor in Western University's department of family medicine.

The WHO's evidence-review process was "clean and thorough," says Lent. The guidelines will be particularly useful to health care providers who feel intimidated or lack the training required to respond to intimate-partner violence, she says.
Because some professional organizations including the Registered Nurses' Association of Ontario and the American Congress of Obstetricians and Gynecologists have endorsed universal screening, many Canadian practitioners have been confused about how to approach intimate-partner violence, agrees Wathen. They also lack training on how to respond to women experiencing violence, she says.

In a survey of university and college health care programs in Ontario that Wathen and colleagues conducted in 2005-2006, they found fewer than half of undergraduate medical and dental programs in Ontario (43\% and 46\%, respectively) offered content addressing intimate-partner violence. At the postgraduate level, nursing schools were more likely to offer this content $(41 \%)$ than medical schools (25\%).

"We need a more robust and consistent approach to training physicians 
when they are in medical school or [nurses in] nursing school, and also to provide ongoing continuing education for those who are out of school," Wathen says. "The WHO guidelines strongly recommend that clinician training be given priority."

The health of women who experienced violence in childhood or are still experiencing it in relationships is "absolutely" affected, says Lent, although not all practitioners recognize to what extent. "Despite the prevalence and despite the depth of the research that has gone on looking at health issues related to intimate-partner violence over the last 10 or 15 years ... it still is not a mainstream issue," Lent says. "We still need to do work to make it more mainstream."

The WHO also released a report indicating a third of women have experienced physical or sexual violence, primarily by intimate partners. The resulting consequences include broken bones, mental health issues, pregnancy-related complications and impaired social functioning. Globally, $38 \%$ of women who were murdered were killed by intimate partners, the report states.

"These findings send a powerful message that violence against women is a global health problem of epidemic proportions," Dr. Margaret Chan, director-general of the WHO, said in a news release. "We also see that the world's health systems can and must do more for women who experience violence."

In her maiden speech to the Canadian Medical Association in August, federal Health Minister Rona Ambrose also urged doctors to address family violence by recognizing the signs, reporting violence and ensuring patients get the physical and emotional support they need. "You are often the first to interact with patients who are dealing with family violence; and research shows that you are the ones victims are most likely to disclose abuse to," she added.

Partner violence has a huge economic impact on Canada, costing an estimated $\$ 7.4$ billion per year, according to an analysis by the Department of Justice released in 2012. The department estimates the health costs alone at $\$ 79$ million per year, a figure that includes physician and emergency department visits, acute hospitalization, mental health services and medical costs associated with treatment of children exposed to family violence. Laura Eggertson, CMAJ

CMAJ 2013. DOI:10.1503/cmaj.109-4609 